\title{
Chaos in multiplicative systems
}

\author{
Dorota Aniszewska $^{1}$ and Marek Rybaczuk ${ }^{2}$ \\ 1 Institute of Materials Science and Applied Mechanics \\ Wroclaw University of Technology \\ 50-370 Wroclaw, Smoluchowskiego 25 \\ (e-mail: dorota.aniszewska@pwr.wroc.pl) \\ 2 (e-mail: marek.rybaczuk@pwr.wroc.pl)
}

\begin{abstract}
The goal of the paper is chaos examination in multiplicative systems. The paper collects results of numerical simulations as well as presentation of methods applicable in the case of multiplicative systems. Chaos examination concerns one-dimensional multiplicative version of logistic equation and multi-dimensional nonlinear system described with multiplicative derivatives. The classical Lorenz system transformed into multiplicative version was chosen for analysis of stability and chaotic behaviour.
\end{abstract}

Keywords: multiplicative calculus, logistic equation, the Lorenz system, Runge Kutta method, Lyapunov exponent.

\section{Introduction}

Main subject of our work is examination if chaos may occur in process of defects evolution in material. Model of fractal defects growth has been presented in Rybaczuk and Stoppel[4]. It is based on single fractal approximation and links the energy uniformly distributed over fractal, its measure $\nu_{D}$ and energy density $a(D)$, which is essential material characteristics depending on fractal dimension:

$$
\mathcal{E}=a(D) \nu_{D}
$$

Appearance of fractals requires changes in the form of physical equations. Mathematical theory of dynamical systems describing physical processes must pay attention to physical quantities. In model (1) shift of fractal dimension means shift of physical dimension. Classic derivative based on differential quotient containing $a(D+\Delta D)-a(D)$ has no physical sense. The labile physical dimension of $a(D)$ is the essential reason for employing multiplicative calculus to formulate dynamical system of defects growth.

This paper collects results of chaos examination for exemplary multiplicative systems. It is expected that presented methods are applicable to a wide variety of multiplicative dynamical systems including dynamical models of fractal defects evolution. 


\section{Multiplicative calculus}

Multiplicative calculus has been presented in Volterra[5]. The differential multiplicative calculus has been restored in Rybaczuk[3], Rybaczuk and Stoppel[4]. Definition of the multiplicative derivative of function $f(x)$ with respect to $x$ follows as:

$$
\frac{\pi f(x)}{\pi x}=\lim _{\epsilon \rightarrow 0}\left\{\frac{f(x(1+\epsilon))}{f(x)}\right\}^{\frac{1}{\epsilon}},
$$

where shift of independent variable $x$ is multiplicative $x \rightarrow x(1+\epsilon)$, therefore $x \in \mathbb{R}_{+}$and $f(x) \in \mathbb{R}_{+}$. In Rybaczuk[3] the generalized methods to examine equations comprising quantities with labile physical dimensions are presented. All notions and theorems of the classic differential calculus have their multiplicative counterparts. To assist further applications we present some of them.

If all subsequent multiplicative derivatives exist, multiplicative derivative of sum and product of functions $f(x)$ and $g(x)$ equals respectively:

$$
\begin{gathered}
\frac{\pi f(x)+g(x)}{\pi x}=\frac{\pi f(x)^{\frac{f(x)}{f(x)+g(x)}}}{\pi x} \frac{\pi g(x)^{\frac{g(x)}{f(x)+g(x)}}}{\pi x} \\
\frac{\pi f(x) g(x)}{\pi x}=\frac{\pi f(x)}{\pi x} \frac{\pi g(x)}{\pi x}
\end{gathered}
$$

The formula for a complex function is more complicated:

$$
\frac{\pi f(g(x))}{\pi x}=\frac{\pi f(g)}{\pi g}^{\ln \frac{\pi f(x)}{\pi x}}=\frac{\pi g(x)}{\pi x}^{\ln \frac{\pi f(g)}{\pi g}}
$$

The function $f(x)$ can have extrema in such points for which:

$$
\frac{\pi f(x)}{\pi x}=1
$$

\section{Lyapunov stability and Lyapunov exponent}

Lyapunov type stability for system of multiplicative differential equations is introduced in Aniszewska and Rybaczuk[2] in details. In this paper it is summarized briefly.

Equilibria of system of autonomous multiplicative differential equations $\frac{\pi x_{j}}{\pi t}=f_{j}\left(x_{1}, \ldots, x_{n}\right)$, where $j=1, \ldots, n$ are obtained from:

$$
f_{j}\left(x_{1}, \ldots, x_{n}\right)=1
$$

Solution close to fixed point $\boldsymbol{x}_{0}=\left(x_{1}^{(0)}, x_{2}^{(0)}, \ldots, x_{n}^{(0)}\right)$ is described by small multiplicative shift $\epsilon_{j}(t)$ :

$$
x_{j}(t)=x_{j}^{(0)}\left(1+\epsilon_{j}(t)\right) .
$$


Behaviour of derivative $\frac{\pi \epsilon_{j}(t)}{\pi t}$ for long time determines if equilibrium $\boldsymbol{x}_{0}$ is stable. Calculation of multiplicative derivatives gives following result:

$$
\epsilon_{j}(t)=\epsilon_{j}^{(0)} t^{\lambda_{j}}
$$

where $\epsilon_{j}^{(0)}$ is determined by initial conditions. Stability of fixed point $\boldsymbol{x}_{0}$ depends on eigenvalues of matrix $\left[\ln \frac{\pi f_{j}\left(x_{0}\right)}{\pi x_{k}}\right]$. For complex eigenvalue stability depends on their real part. If it is negative, for long time $\epsilon_{j}(t) \rightarrow 0$ and $\boldsymbol{x}_{0}$ is stable. If it is positive, value of $\epsilon_{j}(t)$ increases according to power function of time and $\boldsymbol{x}_{0}$ is unstable.

Divergence or convergence of trajectories with close starting points proceeds according to power of time $\epsilon_{j} \approx \epsilon_{j}^{(0)} t^{\lambda}$, therefore Lyapunov exponents for multiplicative dynamical system can be defined as:

$$
\lambda_{j}=\lim _{t \rightarrow \infty} \frac{1}{\ln t} \ln \frac{\epsilon_{j}}{\epsilon_{j}^{(0)}}
$$

and if at least one of them is positive, trajectory behaviour is chaotic.

\section{Multiplicative logistic equation $x_{2 n}=r x^{1-x}$}

The simplest systems employed to chaos examination are one-dimensional ones. Numerical simulations and calculation of Lyapunov exponent have been performed for multiplicative version of logistic equation, which can have a few forms. One of them follows as:

$$
x_{2 n}=\exp \left(r x_{n}\left(1-x_{n}\right)\right),
$$

where $n$ is a step number. In case of multiplicative system, shift of step must be multiplicative: $n \rightarrow n(1+\Delta n)$, where $\Delta n=1$ for discrete system. Therefore following numbers of steps are calculated as $2 n$ equals $1,2,4,8,16, \ldots$. This version of equation is analysed in details in Aniszewska and Rybaczuk[2]. In this paper we present another multiplicative version of the classical logistic equation, which has the form:

$$
x_{2 n}=r x^{1-x}
$$

and for this equation chaos appears for certain range of $r$ parameter values. Phase diagrams and step series for various $r$ values are presented in Figures 1 and 2. For $r=2$ there are only two solutions and for $r=4$ chaotic behaviour appears. Bifurcation diagram is shown in Figure 3(a).

Calculation of Lyapunov exponent is based on two trajectories started from points with distance determined by small multiplicative shift $\epsilon_{0}$. When 


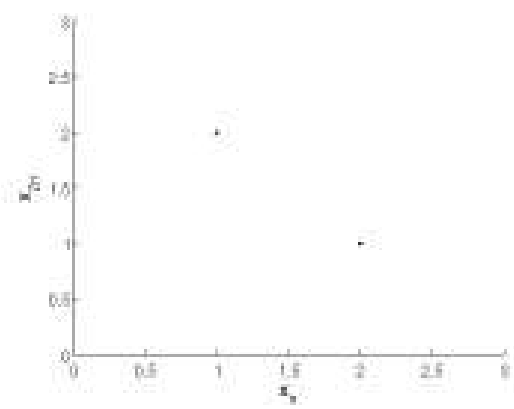

(a)

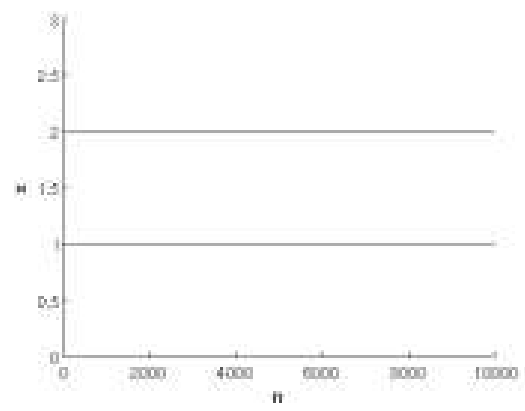

(b)

Fig. 1. Phase diagrams and step series of the multiplicative logistic equation $x_{2 n}=$ $r x^{1-x}$ for $r=2$.

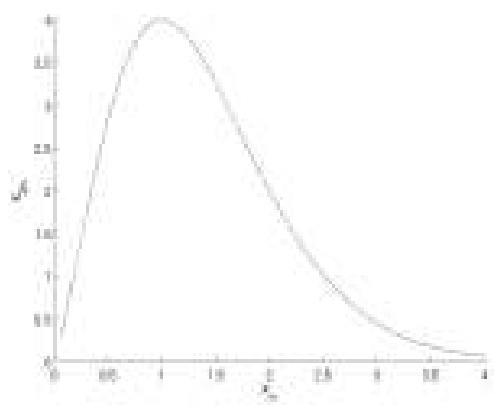

(a)

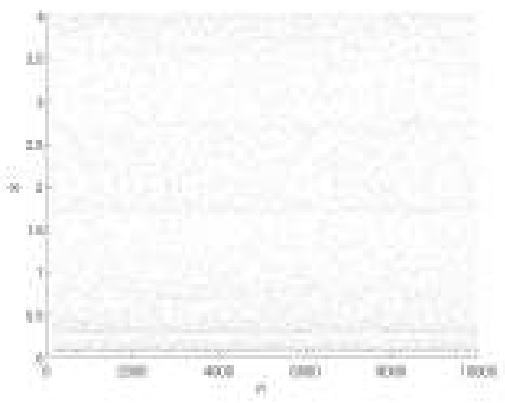

(b)

Fig. 2. Phase diagrams and step series of the multiplicative logistic equation $x_{2 n}=$ $r x^{1-x}$ for $r=4$.

in each $k$ step multiplicative shift $\epsilon_{k}$ between points is calculated, then for many steps we obtain value of Lyapunov exponent:

$$
\lambda=\frac{1}{\ln N} \sum_{k=1}^{N} \ln \frac{\epsilon_{k}}{\epsilon_{0}},
$$

where $N$ is number of steps. Relationship between Lyapunov exponent and $r$ parameter is presented in Figure 3(b).

Slighly different version of multiplicative logistic equation has the form $x_{2 n}=(r x)^{1-x}$. Its behaviour its similar, but chaos appears for other range of $r$ values. Bifurcation diagram for this version is presented in Figure 4(a) and relationship between Lyapunov exponent and $r$ parameter is shown in Figure 4(b). 


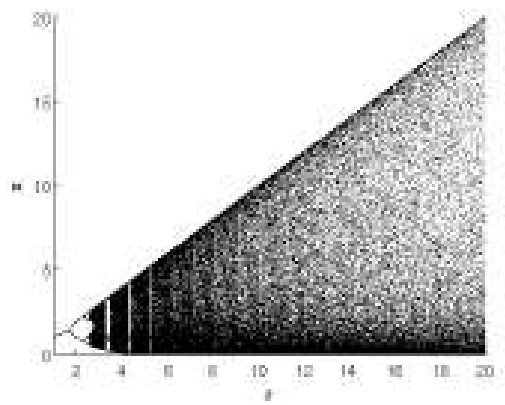

(a)

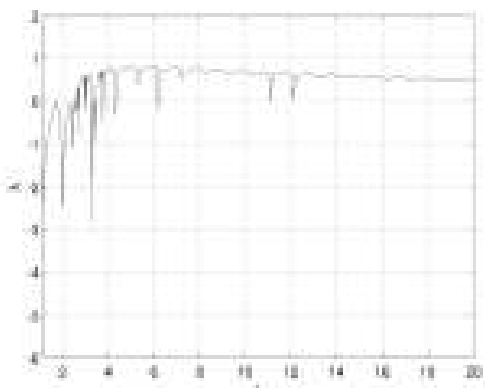

(b)

Fig. 3. Bifurcation diagram for the multiplicative logistic equation $x_{2 n}=r x^{1-x}$ and relationship between Lyapunov exponent and $r$ parameter.

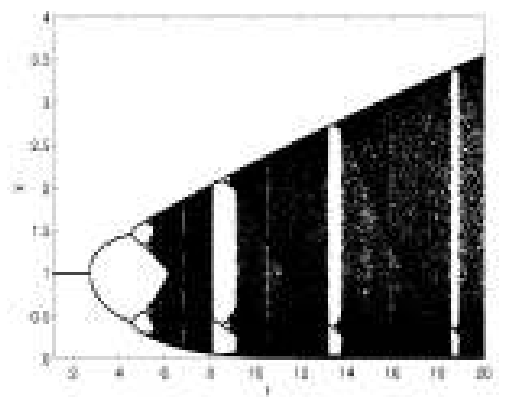

(a)

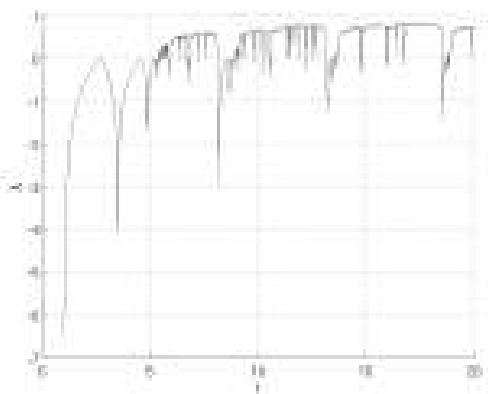

(b)

Fig. 4. Bifurcation diagram for the multiplicative logistic equation $x_{2 n}=(r x)^{1-x}$ and relationship between Lyapunov exponent and $r$ parameter..

\section{Multiplicative Lorenz system}

Main goal of this paper is chaos examination in system described with multiplicative differential equations. The Lorenz system containing three nonlinear equations with three positive real parameters:

$$
\begin{aligned}
& \frac{d x}{d t}=\sigma y-\sigma x \\
& \frac{d y}{d t}=r x-y-x z \\
& \frac{d z}{d t}=x y-b z
\end{aligned}
$$


was chosen as an exemplary chaotic dynamical system and it has been transformed into multiplicative dynamical system: addition was replaced by multiplication and multiplication by raising to suitable power. The multiplicative Lorenz system equals:

$$
\begin{aligned}
& \frac{\pi x}{\pi t}=y^{\sigma} x^{-\sigma} \\
& \frac{\pi y}{\pi t}=x^{r} y^{-1} x^{-\ln z} \\
& \frac{\pi z}{\pi t}=x^{\ln y} z^{-b}
\end{aligned}
$$

Detailed analysis of Lyapunov type stability for this system was presented in Aniszewska and Rybaczuk[2]. It provide the same results as for classical Lorenz system. The solutions of nonlinear multiplicative dynamical systems can be calculated using numerical methods therefore multiplicative RungeKutta methods presented in [1] was employed to perform numerical simulations.

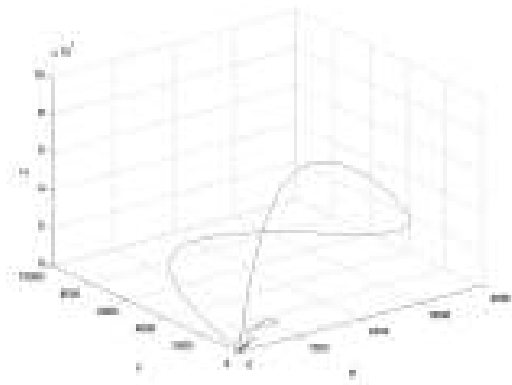

(a) $\mathrm{r}=10$

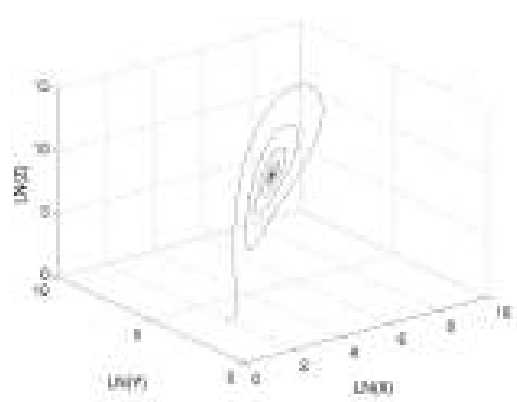

(c) $\mathrm{r}=10$

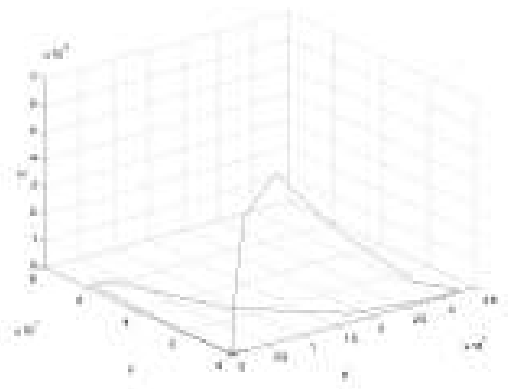

(b) $\mathrm{r}=28$

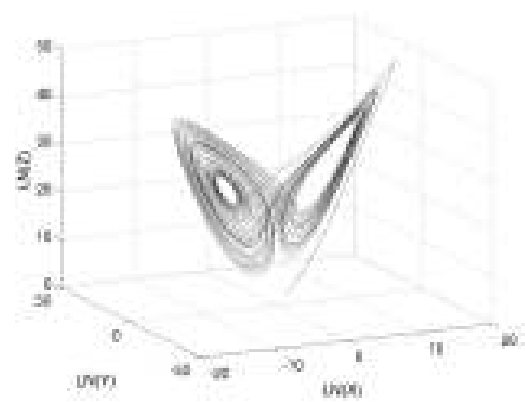

(d) $\mathrm{r}=28$

Fig. 5. Trajectories of the multiplicative Lorenz system (15) for $\sigma=10, b=\frac{8}{3}$ and various $r$ values in linear and logarithmic scale. 
Numerical solutions of the multiplicative Lorenz system (15) revealed that chaos appears for $r>24.84$ for $\sigma=10$ and $b=\frac{8}{3}$. Trajectory spirals out two equilibria and creates butterfly shape attractor, which is visible in logarithmic scale.

In case of multi-dimensional dynamical systems calculating Lyapunov exponents is more complicated, because local behaviour of close trajectories may vary with the direction. For calculation of the largest Lyapunov exponent of the multiplicative Lorenz system we employed multiplicative version of method introduced in [6]. For classical dynamical system the largest Lyapunov exponent equals:

$$
\lambda=\lim _{t \rightarrow \infty} \frac{1}{t} \ln \frac{L(t)}{L(0)},
$$

where $L(t)$ is linear extent of ellipsoid created in space by two trajectories. For multiplicative dynamical systems the largest Lyapunov exponent should be calculated as:

$$
\lambda=\lim _{t \rightarrow \infty} \frac{1}{\ln t} \ln \frac{L(t)}{L(0)}
$$

because time shift is multiplicative.

Results of the largest Lyapunov exponent computation for the multiplicative Lorenz system are given in Table 1.

\begin{tabular}{|l||l|l|l|l|l|l|}
\hline $\mathrm{r}$ & 5 & 10 & 15 & 20 & 25 & 28 \\
\hline \hline$\lambda$ & -0.93 & -0.59 & -0.34 & -0.12 & 0.82 & 0.91 \\
\hline
\end{tabular}

Table 1. The largest Lyapunov exponent of the multiplicative Lorenz system for $\sigma=10, b=\frac{8}{3}, 40000$ steps, starting time 0.01 and time step 0.01.

\section{Discussion}

The results presented in the paper proof that all classical conditions concerning chaotic behavior can be extended onto multiplicative systems. The paper presents exemplary multiplicative systems with chaotic behaviour. Similarly like in case of dynamical system described with classical derivatives, nonlinearity and three indepenent variables are necessary for chaos occuring in systems described with multiplicative derivatives.

\section{References}

1.D.Aniszewska. Multiplicative runge-kutta methods. Nonlinear Dynamics, 50:265 $-272,2007$ 
2.D.Aniszewska and M. Rybaczuk. Lyapunov type stability and lyapunov exponent for exemplary multiplicative dynamical systems. Nonlinear Dynamics, 54:345 $-354,2008$.

3.M.Rybaczuk, A. Kedzia, and W. Zielinski. The concept of physical and fractal dimension II. the differential calculus in dimensional spaces. Chaos, Solitons 83 Fractals, 12:2537-2552, 2001.

4.M.Rybaczuk and P.Stoppel. The fractal growth of fatigue defects in materials. International Journal of Fracture, 103:71-94, 2000.

5.V. Volterra and B.Hostinsky. Herman, Paris, 1938.

6.A. Wolf, J.B. Swift, H.L. Swinney, and J.A. Vastano. Determining lyapunov exponents from a time series. Physica 16 D, pages $285-317,1985$. 